antibody would have untoward side effects. This is certainly possible, because RANK and RANKL have many roles elsewhere in the body. For instance, immune cells called T cells express RANKL, and RANK is found on dendritic cells, with which T cells interact to trigger immune responses; in this setting, the interaction between RANK and RANKL enhances T-cell immunity ${ }^{8}$. The proteins have also been identified in the brain, notably in some neurons and neuron-supporting cells, in brain-specific immune cells called microglia, and in brain tissue deprived of oxygen ${ }^{9}$. They have also been implicated as mediators of fever-related responses to infections ${ }^{10}$.

That said, a decade's worth of clinical studies using denosumab has revealed no discernible effects on the immune system or the brain. There is also no evidence yet for reverse RANK signalling in immune cells or neurons. Nonetheless, given that nearly all nucleus-bearing cells release extracellular vesicles, further studies of RANK-RANKL signalling in non-skeletal tissues are imperative. Given nature's propensity for reusing principles of intercellular signalling in many ways, the possibility of reverse signalling by other receptors must also be explored.

Mone Zaidi and Christopher P. Cardozo are in the Mount Sinai Bone Program, Department of Medicine, Icahn School of Medicine at Mount Sinai, New York, New York 10029, USA.
C.P.C. is also at the James J. Peters Veterans Affairs Medical Center, New York.

e-mail:mone.zaidi@mountsinai.org

1. Zaidi, M. Nature Med. 13, 791-801 (2007).

2. Nakashima, T. et al. Nature Med. 17, 1231-1234 (2011).

3. Xiong, J. et al. Nature Med. 17, 1235-1241 (2011).

4. Ikebuchi, Y. et al. Nature 561, 195-200 (2018).

5. Huynh, N. et al. J. Dent. Res. 95, 673-679 (2016).

6. Singha, U. K. et al. J. Cell Biochem. 103, 434-446 (2008).

7. Zaidi, M., Yuen, T., Sun, L. \& Rosen, C. J. Endocr. Rev. https://doi.org/10.1210/er.2018-00050 (2018).

8. Anderson, D. M. et al. Nature 390, 175-179 (1997).

9. Shimamura, M. et al. Proc. Natl Acad. Sci. USA 111. 8191-8196 (2014)

10. Hanada, R. et al. Nature 462, 505-509 (2009).

This article was published online on 5 September 2018.

\title{
Spins travel far in an antiferromagnet
}

\section{Controlled long-distance transport of electron spins is required for a kind of electronics known as spintronics. Such transport has been realized in an antiferromagnet, the most common type of magnetic material. SEE LETTER P.222}

\section{SERGIO M. REZENDE}

$\mathrm{S}$ trongly magnetic materials called antiferromagnets have an intriguing property. Below a certain temperature, the spins (intrinsic angular momenta) of neighbouring atoms in the material point in opposite directions, so that the material exhibits no response to an external magnetic field. The discovery and pioneering studies of antiferromagnets were made by the physicist Louis Néel, who stated in his 1970 Nobel Lecture that the materials "are extremely interesting from the theoretical viewpoint, but do not seem to have any applications" (see go.nature. com/2lzrly8). This pessimistic view of antiferromagnets would change dramatically a few decades later. Today, the materials have practical applications, and promise to deliver several more. On page 222, Lebrun et al. ${ }^{1}$ report the observation of long-distance spin transport in an antiferromagnetic insulator, demonstrating that such materials could be used for spin-based electronics (spintronics).

The debut of antiferromagnets in technology was made possible through the 1988 discovery of the giant magnetoresistance effect ${ }^{2,3}$, which resulted in the 2007 Nobel Prize in Physics. This discovery showed that, in magnetic structures containing nanometre-thick multilayers (stacks of different ultrathin films), electron transport could be controlled by the spin of the electrons - rather than by their electric charge, as in conventional electronics. This finding triggered research into magnetic multilayers, and gave birth to the field of spintronics, which has revolutionized magnetic recording techniques and promises to bring about advances in information technologies ${ }^{4}$.

Antiferromagnets proved to be essential in sensors that use the giant magnetoresistance effect. Since the late 1990s, the read heads for computer-disk drives have been based on such sensors. These read heads are much more sensitive to changes in magnetic fields than are conventional ones. However, although antiferromagnets are important for spintronic devices, they have had a passive role. Ferromagnets - materials in which all of the atomic spins are aligned - have had the active role.

In the past decade, this situation has begun to change because of experimental and theoretical results showing that antiferromagnets have several advantages over ferromagnets in spintronic devices. One advantage is the insensitivity of antiferromagnets to perturbations in external magnetic fields. Another is their ultrafast dynamics, which could enable devices to operate at terahertz-scale $\left(10^{12} \mathrm{~Hz}\right)$ frequencies and therefore facilitate faster electronics. Developments in the past few years have given rise to the field of antiferromagnetic spintronics $^{5-7}$, which now gains a boost, thanks to Lebrun and colleagues.

The authors used a thin, flat sample of

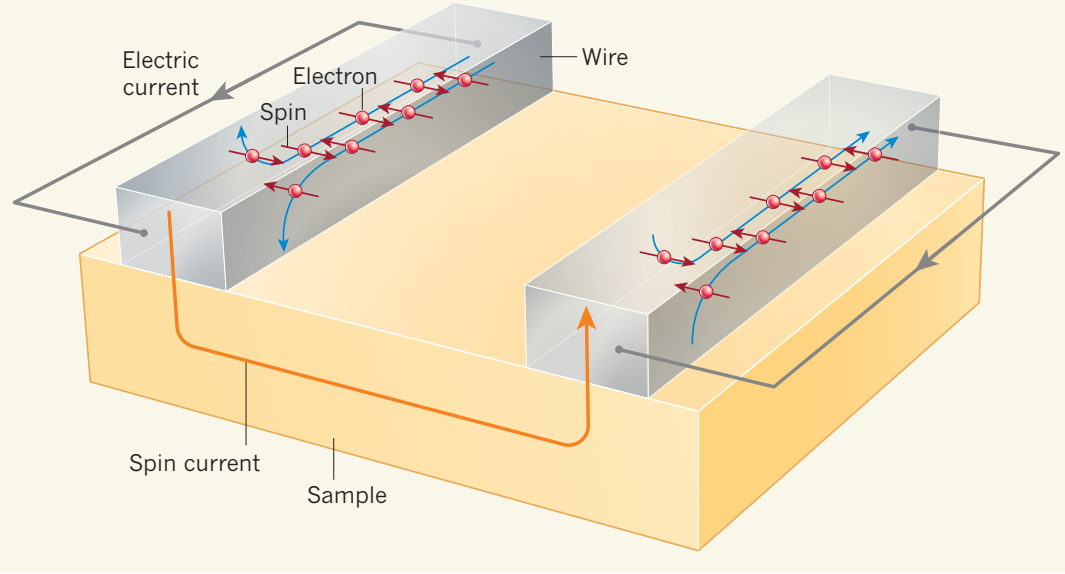

Figure 1 | Spin transport in an antiferromagnetic insulator. Lebrun et al. ${ }^{1}$ carried out an experiment using a thin, flat sample of an electrically insulating material that exhibits a type of magnetism known as antiferromagnetism. The authors deposited two thin platinum wires on the surface of the sample and injected an electric current into one of the wires (the left wire). This electric current was converted into a spin current, which is produced by electrons with opposite spins (intrinsic angular momenta) moving in opposite directions (blue arrows). The spin current flowed into the sample and was transported laterally towards the second wire, where it was converted into an electric current. The authors demonstrated that the spin current could be transported over a relatively long distance between the wires (tens of micrometres at a temperature of 200 kelvin), which is a requirement for spin-based electronics. 
single-crystal haematite, $\alpha-\mathrm{Fe}_{2} \mathrm{O}_{3}-$ an electrical insulator that is the most common antiferromagnetic iron oxide. They deposited two thin platinum wires onto the surface of the sample, such that the two wires were parallel to each other, and injected an electric current into one of the wires (Fig. 1). This electric current was converted into a spin current, which in metals is produced by electrons with opposite spins moving in opposite directions, by means of a phenomenon called the spin Hall effect ${ }^{8}$. The spin current flowed into the sample and was transported laterally towards the second wire, where it was converted into an electric current by the inverse spin Hall effect ${ }^{9}$. Finally, this electric current produced a voltage signal that was picked up by a detector.

By reversing the direction of the injected electric current and calculating the difference in the voltages produced by currents in opposite directions, Lebrun et al. separated the signals arising from the spin Hall effect from those produced by thermal effects. This voltage difference varies linearly with the magnitude of the injected current and can be used to decode information carried by the spin current. The authors measured the voltage difference as a function of several parameters, such as the spatial separation of the wires, the intensity of an applied external magnetic field, and the angle between the direction of this field and the wires.

There are several key achievements in Lebrun and colleagues' work. First, the authors demonstrated spin transport in an antiferromagnetic insulator over a relatively long distance - tens of micrometres at a temperature of 200 kelvin. This finding suggests that such spin transport could be feasible at room temperature, which would be useful for practical applications. The authors argue that antiferromagnets have an advantage over the well-studied ferromagnetic insulator yttrium iron garnet $\left(\mathrm{Y}_{3} \mathrm{Fe}_{5} \mathrm{O}_{12}\right)$, which has been used in similar spin-transport experiments ${ }^{10}$. In devices based on this material, it is not possible to separate the signals produced by the spin Hall effect from those generated by thermal effects.

A second substantial achievement for device application is the authors' demonstration that the flow of the spin current can be controlled by an external magnetic field. The results also resolve a controversial issue: the physical mechanism responsible for spin transport in antiferromagnets. The dependence of the voltage difference on the spatial separation of the wires shows that the spin transport is mainly caused by the diffusion of excitations called magnons ${ }^{11}$, rather than by the spins forming an exotic state of matter known as a superfluid ${ }^{12}$.

Despite these achievements, some obstacles must be overcome before antiferromagnets can be used in practical devices requiring the control of spin currents. One challenge is pointed out by Lebrun and colleagues: finding antiferromagnets in which spins can be transported over even longer distances than demonstrated in the current work. Another, more formidable, challenge is to find materials amenable to spin-current control using weaker magnetic fields than those used by the authors. Nevertheless, the current findings provide additional impetus to the emerging field of antiferromagnetic spintronics.

Sergio M. Rezende is in the Department of Physics, Federal University of Pernambuco, 50670-901 Recife, Pernambuco, Brazil. e-mail:rezende@df.ufpe.br

1. Lebrun, R. et al. Nature 561, 222-225 (2018).

2. Baibich, M. N. et al. Phys. Rev. Lett. 61, 2472-2475 (1988).
3. Binasch, G., Grünberg, P., Saurenbach, F. \& Zinn, W Phys. Rev. B 39, 4828-4830 (1989).

4. Waldrop, M. M. Nature 530, 144-147 (2016).

5. Jungwirth, T. et al. Nature Phys. 14, 200-203 (2018).

6. Baltz, V. et al. Rev. Mod. Phys. 90, 015005 (2018)

7. Jungfleisch, M. B., Zhang, W. \& Hoffmann, A. Phys. Lett. A 382, 865-871 (2018).

8. Hirsch, J. E. Phys. Rev. Lett. 83, 1834-1837 (1999).

9. Saitoh, E., Ueda, M., Miyajima, H. \& Tatara, G. Appl. Phys. Lett. 88, 182509 (2006)

10.Cornelissen, L. J., Liu, J., Duine, R. A., Ben-Youssef, J. \& van Wees, B. J. Nature Phys. 11, 1022-1026 (2015).

11.Rezende, S. M., Rodríguez-Suárez, R. L. \& Azevedo, A. Phys. Rev. B 93, 054412 (2016).

12.Takei, S., Halperin, B. I., Yacoby, A. \& Tserkovnyak, Y. Phys. Rev. B 90, 094408 (2014).

\title{
A 3D cell shape found in sheets and tubes
}

\begin{abstract}
Sheets of cells called epithelia can curve into tubes in embryos. Modelling and in vivo observations reveal that cells in tubes adopt an asymmetric cell shape dubbed scutoid, contrary to some previous assumptions.
\end{abstract}

\section{GUY B. BLANCHARD}

$\mathrm{E}$ mbryo and organ development is driven in large part by the sculpting of sheets of cells called epithelia. Epithelial sheets have a remarkable repertoire of behaviours they can narrow and elongate in plane, change thickness, and bend out of plane to form structures such as pits and furrows that develop into the body's tubes. However, our understanding of the $3 \mathrm{D}$ shapes of the cells that make up these structures, and of how they change during development, has been dominated by inferences often made from looking only at the most easily imaged surface of an epithelial-cell sheet. Writing in Nature Communications, Gómez-Gálvez et al. ${ }^{1}$ use a modelling approach to predict how cells are packed to form tubes, and the authors give a name to a type of epithelial-cell shape involved in this packing, which they also find occurs in vivo.

Cells in single-layered epithelia have approximately parallel tops (apices) and bases. The cells are therefore usually thought of as prisms that join together in a honeycomb-like arrangement (Fig. 1a). When either the apices or the bases of cells constrict in all directions (isotropic constriction), wedge-shaped cells called frusta emerge (Fig. 1b), resulting in the formation of a pit $^{2}$ or furrow ${ }^{3}$.

During the elongation of fruit-fly embryos, cell-cell junctions at the apices ${ }^{4}$ or bases ${ }^{5}$ of epithelial sheets can also contract in just one orientation in the plane of the sheet. This anisotropic constriction can lead to the formation of different contacts between neighbouring cells at the apical end of the cell from contacts at the basal end. Such constriction produces cells that are neither prisms nor frusta - but the shape that they form has had no name.

Gómez-Gálvez et al. analysed 3D cell shapes in epithelia by using computer modelling to study how cells pack into curved epithelia. For epithelia on a sphere, surface curvature is the same in all directions, and inverted frusta are expected. But the authors realized that, when epithelia form tubes and so curve in only one orientation, or form egg shapes that also have differential surface curvature, the cells' inner and outer surfaces can contact different neighbours. This change in contacts requires the formation of a triangular face on one side of the cell (Fig. 1c) - a 3D shape similar to that seen in the epithelial sheets of elongating fruit-fly embryos. The researchers name this $3 \mathrm{D}$ cell shape scutoid, from the Latin word for shield.

So why are scutoids predicted to occur in tubes? It is possible for $3 \mathrm{D}$ shapes resembling frusta to form tubes. But this requires the cells' apical or basal surfaces to be elongated, and Gómez-Gálvez et al. based their modelling on the plausible assumption that apical and basal surfaces of epithelial cells both tend towards isotropic shapes as the most energy-efficient ('relaxed') packing solution to differential curvature. This is in contrast to the sheets of fruit-fly embryos, in which the formation of scutoids is a dynamic, temporary process driven by active cell rearrangement ${ }^{5}$. When the authors modelled cells that had near-isotropic shapes on both the inner and outer surfaces 